\title{
Analysis of the Modeling Method and Application of 3D City Model based on the Cityengine
}

\author{
Xifang $\mathrm{JIN}^{1, \mathrm{a}}$, Fangzheng WANG ${ }^{2, \mathrm{~b}}$, Lianxiu $\mathrm{HAO}^{2, \mathrm{~b}},{ }^{\text {, }}$ aping $\mathrm{DUAN}^{2, \mathrm{~b}}, \mathrm{Lili}$ \\ $\mathrm{CHEN}^{2, b}$ \\ ${ }^{1}$ North China Sea Marine Forecasting Center of Station Oceanic Administration, North China Sea \\ Branch of State Oceanic Administration, Qingdao, 266061,China \\ ${ }^{2}$ College Of Geomatics, Shandong University of Science and Technology, \\ Qingdao, 266590,China \\ aemail:419958059@qq.com, bemail:407615037@qq.com
}

Keywords: CityEngine; 3D modeling; CGA rules

\begin{abstract}
Modeling method at present based on traditional manual modeling method has bottleneck problems that restrict the development of digital city, mainly for the long cycle, large workload, high cost. Based on the analysis of digital city and 3D GIS, this paper designs a solution based on CityEngine 3D modeling and studies how to make the modeling efficient and batched modeling by procedural rules, and summed up a set of general-purpose modeling technique process. On this basis, the paper analyses the existing problems in the modeling process combined with actual cases, gives corresponding solutions from the aspects of map data processing v building texture acquirement and model expression optimization, at last this paper carries on 3D modeling with Tangdao Bay area of Qingdao as a demonstration area.
\end{abstract}

\section{Introduction}

As the basis for the construction of digital city scene,3D modeling technology provides city managers and planners with an intuitive and stereoscopic visual perception. At the same time, it has a very important significance for the city's information construction [1]. With people's continuously increasing attention, 3D GIS technology has been rapidly developed. Some domestic cities have already started building digital city systems based on 3D GIS technology [2].

With the expansion of cites, the data amount of modeling is also in the rapid growth, the modeling efficiency becomes a technical bottleneck of the development of 3D city. The traditional manual modeling method can display a realistic 3D city scene, but it must invest very large funds, manpower and material resources [3], and the results are often "constant" and "static". By modeling in this way, the relationships between the model and spatial database did not establish, and it also does not match for the design rules. Although the browse function can be realized, it is very difficult to achieve the subsequent function including calculation for area and volume or other statistical analysis. Therefore, it is also very difficult to have a fully function in the city planning design, audit and supervision. So the traditional modeling methods have been unable to meet the needs of users [4].

This paper mainly studies the modeling method of ESRI's 3D modeling software CityEngine, presents an efficient and rapid modeling technical process using CityEngine, and by combining with the practical application, it gives respectively solutions for the problems of the modeling process.

\section{Analysis of CityEngine modeling process}

CityEngine has a unique way of modeling and uses the parameterized CGA rules to create the model, and can generate batch [3]. CityEngine is a part of ESRI ArcGIS software system, therefore, we can use existing GIS spatial data in the modeling process, and associate with attribute information of GIS data to improve the efficiency of 3D City Modeling[5][6]. 
This paper takes an example of the 3D modeling for Tangdao Bay Area in Huangdao District of Qingdao, the modeling process was summarized as Figure 1.

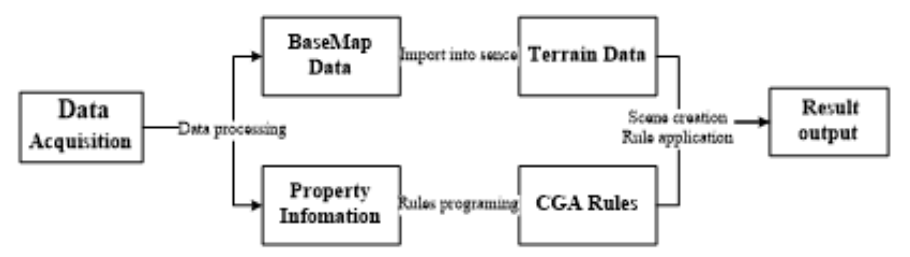

Fig.1. Modeling flow chart

The data collection mainly includes the terrain data such as DEM, images, line drawing, etc and the attribute data like floor height, external texture etc. The terrain data needs a process of matching and superposition; attribute data needs to be standardized and associate with vector data; then construct rules and realize the building of batch modeling.

A. Data collection

Data collection is the preparatory work of modeling, which needs not only to collect the relevant data, but also to process according to the later modeling requirements. From the perspective of data processing, this paper divided the data into two types: basic geographic data and texture data.

a) The basic geographic data

The basic geographic data mainly includes major buildings' map vector data such as images, DEM, buildings, roads and etc. of the modeling areas and other building attribute information and so on. The process of the main work is as shown in Figure 2 (a).

The modeling area's images and DEM data need to re-sized, unify coordinate system and elevation, in order to converted into TIFF format, and then that can be loaded into the scene as the terrain layer.

The modeling area's green point data, road line data, and housing plots numbers are generally gained by purchasing digital surveying and mapping products. Its attribute information includes the floor numbers, roof style, materials, width of road and name of building, which is obtained by collecting related information or field investigation, and associate with the vector data to form $2 \mathrm{D}$ vector data.

The elevation information of $2 \mathrm{D}$ vector data is needed to match and to be added to terrain layer. The data are generally obtained by DEM interpolation. This paper adopts ARCGIS and its related tools for processing. Point element interpolates (Interpolate Shape tools) directly based on DEM, otherwise, line elements need to be simplified (simplify line tools) first, then to interpolate. The dealing process of surface elements are relatively complex: firstly, turn the surface elements into point elements, and obtain elevation by interpolation in point format; then transfer the elevation attribute information to the original surface by linking; finally, realize the $3 \mathrm{D}$ transformation of surface elements with ArcGIS 3D Assignment Tool. The above process is as shown in Figure 2(b):

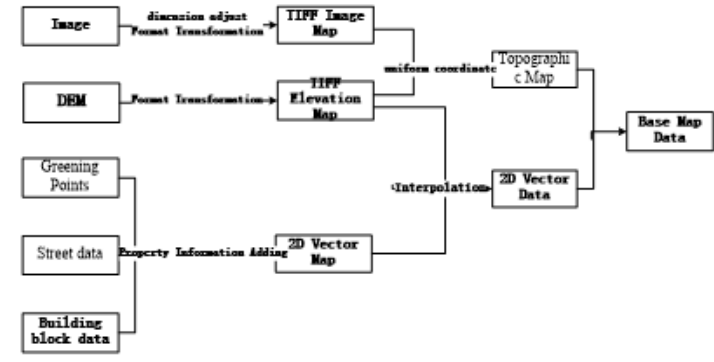

(a) Acquisition and processing of geographic data

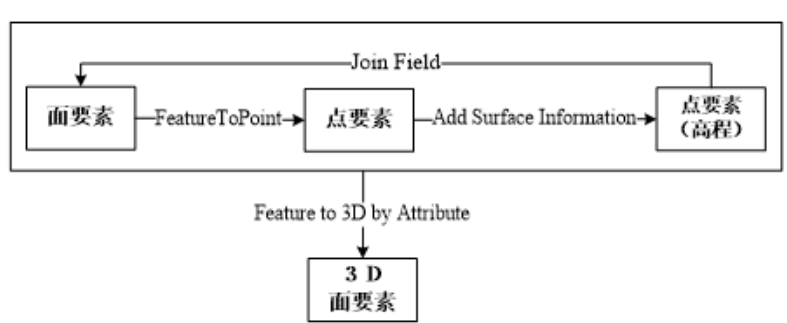

(b) 2D To 3D of the surface element

Fig.2. Some Flow Charts

Through the above process, data of DEM, image and vector have a uniform spatial reference and elevation information, and can be loaded into a CityEngine scene as the basic geographic data.

b) The texture data

The texture data, which also called texture mapping, mainly refers to the surface's color pattern of the modeling object. Acquisition and processing of texture data of 3D model is a key link in 3D 
modeling, which plays an important role in the overall effect of the scene and the display of the model's detail, and is usually processed through Photoshop.

This paper collects materials by field reconnaissance of Tangdao Bay Area. Since texture data by field collection is not uniform in the aspects of resolution, angle, size, color and etc., they often cannot meet the demand of texture mapping of 3D model, so a process of original data is needed. This paper chooses Photoshop as the image processing tool to process the original texture data, including image splicing, cutting, correcting, sundry eliminating, color adjustment, resolution adjustment and etc. For subsequent rule invocations, the processed texture data after processing needs to be named in a uniform way. After the above process, the ortho texture data set which meets requirements for modeling can finally get.

B. Rule analysis

Procedural automatic modeling of CityEngine is based on CGA (Computer Generated Architecture) rules, defines a series of detail rules for how to generate models, and more and more close to the iterative evolution model [8]. The model is generated when map vector data calls the CGA rules file.

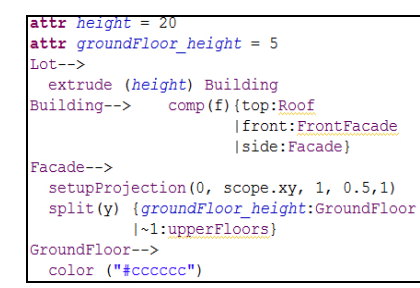

(a) sample of rules writing

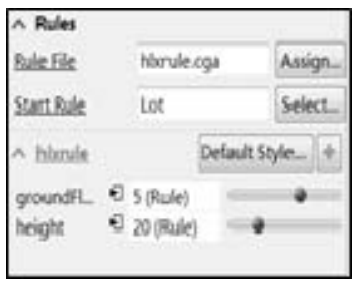

(b) window to view the properties

Fig.3. Samples of basic rules

As is shown in Figure 3 (a), this is one basic CGA rule, including the definition of attribute information, the start rule, model segmentation rules and specific mapping method of texture. A general 3D body can be obtained by this rule, and endowed with texture. As the parameterized rule writing mode of CityEngine, the model parameters can be directly modified during the process of adjusting model details, to realize the interactive adjustment of model, as is shown in Figure 3 (b), groudFL represents first floor height, height represents the whole building height, these properties can be adjusted interactively.

CityEngine creates the model relying on the base map elements, and the basic elements are not necessarily regular shape, then when call the same rules file, the CGA rules will generate model adaptively based on the base map, as is shown in Figure 4.The base map's shape are not the same, but can be applied to the same rules, generative models can reflect the characteristics of the base map, and this achieved mass modeling and rules reusing, and was essentially different from the traditional modeling copy.
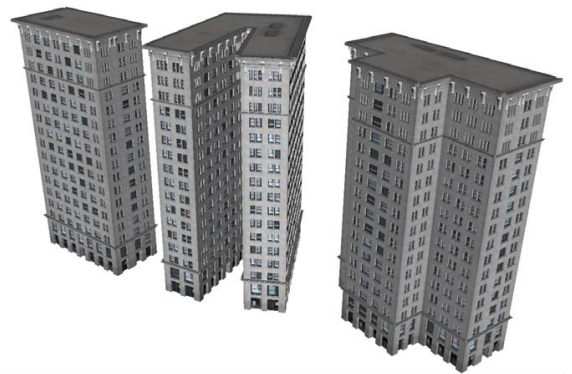

Fig.4. Adaptive Model Generation

\section{The key problems and Solutions}

\section{A. Common data problems}

There are common data problems including the superimposition of terrain and image and display problems of map vector data in the modeling process of CityEngine.

a) Superimposition of terrain and image 
Problems that image or terrain does not display or abnormal display often appear when the image and terrain data is imported to CityEngine. This is mainly because image and terrain size do not meet requirements or pixel depth mismatches. Check and modify the file as the steps below:

(1) Check the size of image and terrain data. CityEngine has some limits on the size of the image and terrain data that the rows and columns of a single data required to be within $8192 * 8192$. If exceed, image and terrain could processed by resampling or cutting.

(2) Check the pixel depth. The pixel depth of DEM in CityEngine is 8 bit, and if conflict, convert the DEM storage format into 8 bits.

b) Scene data spatial reference problems

In CityEngine, models are created based on the actual spatial reference including terrain image coordinate, base map vector data coordinate and the scene coordinate. When the three coordinate systems do not match or the spatial location's deviation is too far, you are prompted that the data is too far to be loaded normally. Data need to be dealt with before they are imported into scene. The steps are shown as follows:

(1) Load data into ArcMap to check whether the data location is correct;

(2) Check the Spatial reference information of data. CityEngine only supports the projection coordinate system at present so if the reference information is "UnKnown", we needn't to choose coordinates when creating the scene;

(3) Check whether image or terrain data have coordinate information file. Data must have coordinate information named as *.tfw file; otherwise, we must correct it through a GIS projection tool.

B. The data extraction of roof texture

Extracting roof texture and matching vector data rapidly is a key problem to be solved in this paper. This issue can be summarized as the following three key technical points:

(1) Get the corresponding slice images of roofs according to the image data in modeling region;

(2) Problem of attribute fields adding that map vector data required;

(3) Retrieve and call building roof texture data in CGA rules,;

(4) Problems of rules writing;

We need to determine the coordinates and dimensions of corresponding image starting point of each element of vector data after the element was cut in stage(1) (3), and realize the image cutting and naming based on each vector element. In order to simplify the corresponding work flow, this paper cites an ArcGIS tool of image cutting. Firstly, get the building plane contours based on the $2 \mathrm{D}$ vector data, cut the corresponding region from image and save it as a picture by image cutting tools based on shape of ArcGIS, and this picture is the roof texture of the model.

In order to add texture to model accurately, texture starting coordinates of the texture need to be recorded, this paper adds 5 fields to the property sheet, using $\mathrm{X}, \mathrm{Z}$ to record starting level coordinates, Xrange, Zrange to record texture range ,Material to record texture name. As is shown in Figure $5(\mathrm{a})$ :

\begin{tabular}{|r|l||c|c|c|r|r|}
\hline FID & Shape & Material & X & Z & Xrange & Zrange \\
\hline 0 & 面 & ImgClip_0 & 24430 & 39811 & 83 & 68 \\
\hline 1 & 面 & ImgClip_1 & 24435 & 39810 & 82 & 67 \\
\hline 2 & 面 & ImgClip_2 & 24451 & 39811 & 78 & 61 \\
\hline 3 & 面 & ImgClip_3 & 24453 & 39811 & 65 & 52 \\
\hline
\end{tabular}

(a) attribute fields added
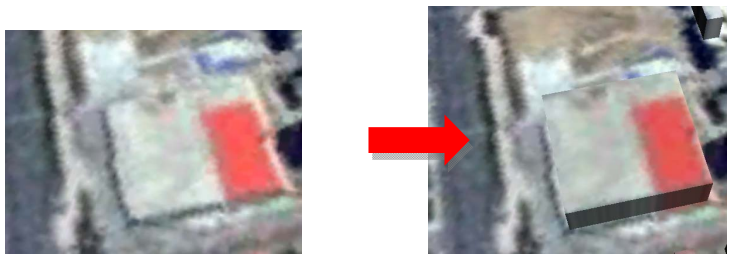

(b) Effect of Roof Texture Extraction

Fig.5. Roof Texture Extraction

By this treatment, the roof texture can match the background map better.As is shown in Figure 5 (b).

C. Model optimization problems

Usually the more precise the model is, the more complex it is, and the more memory it occupy, resulting in the reduction of display speed. Complexity of model reflected in the number of surface and the node of model. cleanupGeometry rule function, Cleanup Shape tool as well as the LOD 
mechanism in CityEngine can optimize the model by classification adapting to improve the display efficiency.

a) cleanupGeometry rule function

In the process of modeling, we often import some fine external models or components. As they occupy too much memory, we need to optimize the processing. The number of nodes and surface will be reduced effectively by importing external models by rules into CityEngine and using cleanup Geometry rule function or Cleanup Shape tool to optimize the model, as is shown in Figure 6:

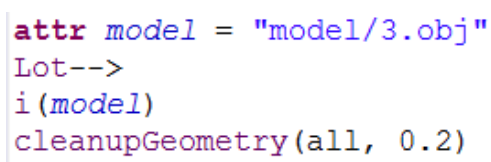

Fig.6. Model optimization rule

\section{b) the LOD mechanism}

LOD (Levels of Detail) is a multi-detail level mechanism, mainly according to the scene display requirements, setting different detail levels of building model, on the premise of not affecting the display effect of the 3D scene, to reduce memory consumption, so that the 3D scene can display smoothly.

In this example, we set up two display levels, taking the window component of model for instance, you can see the LOD property in the properties view window.

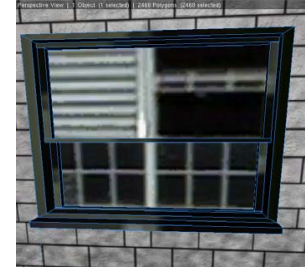

(a) $\mathrm{LOD}=1$

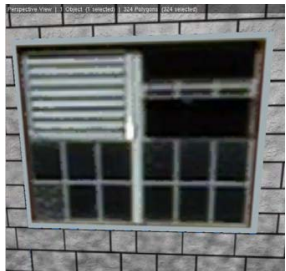

(b) $\mathrm{LOD}=0$

Fig.7. comparison diagram of model optimization

When the LOD parameter equals to 1 , as is shown in Figure 7 (a), rule calls the relatively sophisticated model, the details of the model shows that there are 2466 planes, and the model is more sophisticated, such as window projecting, the effect will be very lifelike in close models show.

When the LOD parameter value is 0 , as is shown in Figure 7 (b), the details of the model are shown in the following: 324 planes. Then the CGA rules directly paste a planar texture on the model, only using very little memory, in a wide range of 3D scene, the display efficiency is relatively fast.

\section{Conclusion}

This paper analyzes the related problems based on CityEngine modeling, gives the corresponding solutions, and forms a comparatively feasible modeling process. Based on the scheme proposed in this paper and using Tangdao Bay community as the modeling demonstration area, the modeling efficiency has greatly improved when we use CityEngine to create 3D models combined with GIS data. Figure 8 is the final effect diagram.

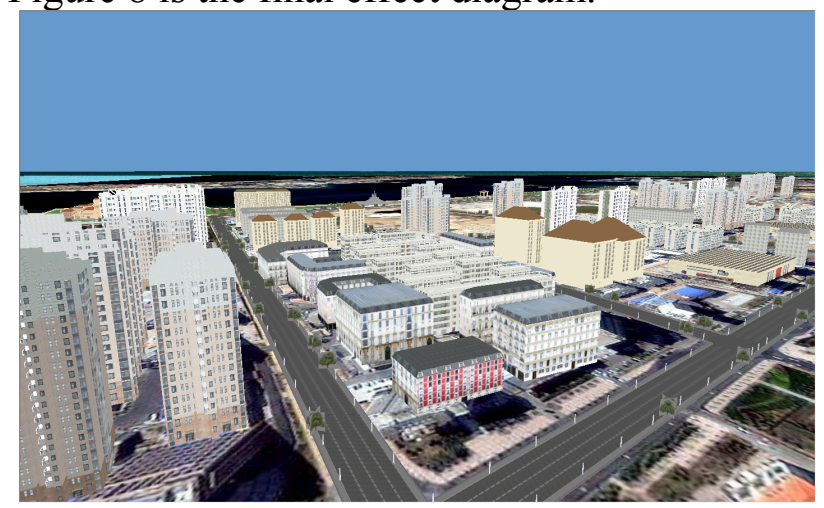

Fig.8. Tangdao Bay effect diagram 


\section{Acknowledgement}

This paper are supported by Public science and technology research funds projects of ocean of which project number is 201205010-4, and of which project number is 201105016-4. We would like to express appreciations to colleagues in our laboratory for their valuable comments and other helps.

\section{Reference}

[1] Deren Li, Jianya Gong, Zhenfeng Shao. From Digital Earth to Smart Earth[J]. Geomatics and Information Science of Wuhan University, 2010,35(2):127-132.

[2] Weisen Li. On Innovation of Establishment of Digital City Geospatical Framework[J]. Bulletin of Surveying and mapping. 2011,9:1-4

[3] Chengming LI, Xiaoli LIU, Jie YIN, Investigation and Practice of Digital City to Smart City[J]. Bulletin of Surveying and mapping.2013,3:1-3

[4] Hanwei Xu, Xiaoliang Fang, Jiayong Ren.3D modeling technique of digital city based on SketchUp[J]. SCIENCE OF SURVEYING AND MAPPING,2011,36(1):213-214

[5] Yongquan Li, Wenquan Han, Zhizhou Huang .Analysis of Means to Build Digital Three Dimensional City Models[J].Modern Surveying and Mapping,2010,33(2):33-35

[6] Jing Wu, Fengxiang Jin, Jian Wang. Three dimension modeling of buildings based on three dimension laser scanner data $[\mathrm{J}]$. ENGINEERING OF SURVEYING AND MAPPING,2007.10,16(5):57-60

[7] Yong Li, Xiangyang Hao, Qin Xi.The Research of some Technologies about 3D-GIS[J]. SCIENCE OF SURVEYING AND MAPPING, 2001(4):25-28+1.

[8] Juan Li, Xi Liu, Jieyu Han. Three dimensional city design method study based on parameterization modeling[J].Urban Managerial Technology, 2013(3):66-68

[9] Esri (China) Information Technology Co., Ltd. CityEngine Chinese tutorial [M]. 2012.

[10] Nian Xie, Yu Xiang, Tingwei Xu. Research on Rapid 3D Modeling Technology Based on Rules[J]. Urban Geotechnical Investigation \& Surveying.2013,4:5-8

[11] Yonglai Lv, Xiaol Li. Exploration and Realization of 3D Modeling Method Based on CityEngine for High-speed Railway. Mapping.2013,36(1):19-22 